Original Research Article

\title{
A prospective study of efficacy and safety of olopatadine versus azelastine in allergic conjunctivitis at a tertiary care hospital
}

\author{
Jayanthi C. R., Nanthini R.*, Vijayalakshmi
}

Department of Pharmacology, Bangalore Medical College and Research Institute (BMC\&RI), Bengaluru, Karnataka, India

Received: 17 May 2017 Accepted: 24 June 2017

*Correspondence to:

Dr. Nanthini R.,

Email: drnandhu89@gmail.com

Copyright: (C) the author(s), publisher and licensee Medip Academy. This is an openaccess article distributed under the terms of the Creative Commons Attribution NonCommercial License, which permits unrestricted noncommercial use, distribution, and reproduction in any medium, provided the original work is properly cited.

\begin{abstract}
Background: Allergic conjunctivitis, an ocular surface inflammatory disease with significant social and economic impact affects approximately $25 \%$ of the general population. $\mathrm{H} 1$ receptor blockers, mast cell stabilizers and drugs that block cytokine and prostaglandin formation form the treatment armamentarium. Olopatadine hydrochloride and Azelastine hydrochloride are dual-acting selective $\mathrm{H} 1$ receptor antagonist with mast-cell stabilizing property. This study was undertaken to assess the efficacy and safety of olopatadine hydrochloride $0.1 \%$ and Azelastine hydrochloride $0.05 \%$ in allergic conjunctivitis amongst Indians.
\end{abstract}

Methods: After obtaining Institutional Ethics Committee approval and written informed consent, 120 patients diagnosed with allergic conjunctivitis fulfilling the inclusion/exclusion criteria were enrolled in the study. Study was done from April 2014 to June 2015 at Minto eye hospital. Study subjects were treated with olopatadine hydrochloride $0.1 \%$ and Azelastine hydrochloride $0.05 \%$ eye drops BD for 15 days. Ocular symptoms, instead of and signs and adverse events, if any were recorded on the day 8 and day 15 follow up visits.

Results: At the end of the study period, the reduction in the ocular itching score from baseline was higher in the olopatadine group compared to the azelastine group $(\mathrm{p}<0.002)$. Similarly, the scores of ocular congestion $(\mathrm{p}<0.008)$, foreign body sensation $(\mathrm{p}<0.009)$, tearing $(\mathrm{p}<0.001)$, erythema $(\mathrm{p}<0.002)$ and chemosis $(\mathrm{p}<0.015)$ also showed larger reduction in the olopatadine treated patients. The common adverse events encountered in both the groups were stinging after instillation, burning, bitter taste and headache.

Conclusions: In allergic conjunctivitis, both olopatadine and azelastine were found to be effective in relieving ocular signs and symptoms, but olopatadine was found to be superior in terms of efficacy, safety and tolerability with minimal side effects.

Keywords: Allergic conjunctivitis, Azelastine hydrochloride $0.05 \%$ eye drops, Olopatadine hydrochloride $0.1 \%$ eye drops

\section{INTRODUCTION}

Allergic Conjunctival Diseases (ACD) are ocular disorders caused by allergic inflammation of the ocular surface and include Allergic conjunctivitis (AC), atopic keratoconjunctivitis (AKC), vernal keratoconjunctivitis (VKC) and giant papillary conjunctivitis. Allergic conjunctivitis is an ACD in which the conjunctiva shows no proliferative change and includes seasonal and perennial allergic conjunctivitis. About $15-20 \%$ of the population worldwide is affected by seasonal allergic conjunctivitis (SAC) which is a type 1 allergic reaction. ${ }^{1}$ SAC has a significant impact on the social and economic aspects of life by increasing health costs and number of disability days leading to absence from work and school. ${ }^{2,3}$

The principal symptom of Allergic Conjunctivitis is ocular itching. Other signs and symptoms include conjunctival redness, tearing, mucus discharge, chemosis and lid edema. Mast cells play an important role in the pathogenesis of Allergic Conjunctivitis. The binding of 
specific antigens on mast cells in the conjunctiva leads to mast cell degranulation and the release of mediators including histamine, tryptase, leukotrienes, cytokines and platelet activating factor. Histamine, being the principal vasoactive amine, is said to be responsible for the major signs and symptoms of allergic conjunctivitis., ${ }^{4,5}$

The most effective means of managing allergic conjunctivitis is to identify the offending antigen and avoid exposure to it. However, on most occasions either the antigens are unidentifiable or exposure cannot be avoided as they are airborne, making this approach implausible. ${ }^{6}$ Thus pharmacological agents like NSAIDs, $\mathrm{H}_{1}$ receptor blockers, mast cell stabilizers and drugs that block cytokine and prostaglandin formation form the treatment armamentarium. Topical antihistaminics, because of their ability to provide rapid relief of signs and symptoms, are the first line agents in the treatment of ocular allergic disorders, but should not be used for more than six weeks. On the contrary, Mast cell stabilizers carry prophylactic value i.e., they prevent future exacerbations but take 3-7 days to show an effect. They can also be used for longer periods when necessary. ${ }^{7}$ Recently, dual action anti-allergic drugs having both antihistaminic and mast cell-stabilizing properties have been introduced which attack multiple facets of the allergic cascade. Olopatadine hydrochloride $0.1 \%$ is one such single molecule with dual properties inhibiting immediate hypersensitivity reactions and providing long term membrane stabilization. ${ }^{8}$ Azelastine, with similar properties, provides immediate relief and the early-phase intervention inhibits expression and activation of inflammatory mediators which characterize the late phase of the immune reaction. ${ }^{9}$ Both have been reported to be effective and well tolerated in the treatment of allergic conjunctivitis but the limited body of evidence amongst Indian population prompted us to evaluate the efficacy and safety of these two ocular preparations. The objective of this study was to compare the reduction in the ocular signs and symptoms scores, safety and tolerability of olopatadine hydrochloride $0.1 \%$ eye drops versus Azelastine $0.05 \%$ eye drops in the treatment of allergic conjunctivitis in the Indian population.

\section{METHODS}

This was a prospective, randomized, open labelled study conducted at outpatient department of Minto Ophthalmology Hospital attached to Bangalore Medical College and Research Institute between April 2014 and June 2015. Patients of either sex aged 18-65 years diagnosed with allergic conjunctivitis with grade 4 (severe) ocular itching and grade 2 or higher (i.e., moderate to severe) bulbar conjunctival injection were included in the study. Patients not willing to give written informed consent, pregnant and lactating women, patients with known hypersensitivity to the study medications or their components (including benzalkonium chloride) and those on other medications which may interfere with the study like decongestants, topical prostaglandin derivatives, ocular or systemic NSAIDs and corticosteroids were excluded from the study. Also not included in the study were patients with an intraocular pressure of more than $21 \mathrm{~mm} \mathrm{Hg}$ in either eye, those having active ocular infection, serious ocular pathological conditions or have undergone ocular surgical interventions within past 3 months and patients unwilling to discontinue wearing contact lenses during the study.

After obtaining informed consent, 120 eligible patients were randomized using a computer generated random number table in a 1:1 ratio. Sixty patients were allocated to each group to receive either olopatadine hydrochloride $0.1 \%$ eye drops $12^{\text {th }}$ hourly or Azelastine hydrochloride $0.05 \%$ eye drops $12^{\text {th }}$ hourly for 15 days. Demographic data, ocular and medical history, concomitant medications, physical examination, clinical examination including recording of vital signs, ophthalmological examination (ocular sign and symptom score) and details of drug prescription were recorded in the study proforma at baseline visit (visit 1) and all subsequent follow-up visits. $^{10-13}$

Follow-up visits were done on day 8 (visit 2) and day 15 (visit 3) after administering the study drugs. A deviation of \pm 1 day for first follow-up and \pm 2 days for subsequent follow-up was accepted. At each follow-up visit data on concomitant medications, ocular symptoms such as itching, foreign body sensation, stinging, photophobia, watering was graded by the patients on a severity scale of 0 to 4 .

Similarly, ocular signs such as congestion, erythema, chemosis was graded by ophthalmologist on a severity scale of 0 to 4 . Safety was assessed by recording the adverse effects at each follow up visit. Study medications were dispensed once at baseline visit. During the study period, subjects were not allowed to take any other topical anti inflammatory drugs except for lubricating eye drops.

A reduction in the ocular signs and symptoms score was the primary efficacy parameter. Clinical Global Impression of Change (CGIC) and Patient Global Impression of Change (PGIC) were the secondary efficacy parameters observed. Safety monitoring was done continuously throughout the study. All adverse effects spontaneously reported by the subjects or elicited by the investigators were recorded.

Tolerability of the study medications was assessed by administering the Global Evaluation of Overall Tolerability (GEOT) scale to the patients which was graded as excellent, good and poor. Compliance was assessed with the help of a medication compliance card. Non responders were defined as those who failed to respond to the trial medication i.e. no reduction in the baseline ocular signs and symptom score at day 8 (visit 1) and thereby requiring additional medications such as topical steroids and immunosuppressants. 


\section{RESULTS}

\section{Demographic characteristics}

Both the study groups were age and gender matched. The mean age of the study participants was 23.3 years.

\section{Efficacy parameters}

Table 2 and Table 3 show the scores for allergic conjunctivitis signs and symptoms at each visit.
There were no significant differences among the groups regarding baseline scores. At each visit, in both groups, scores for ocular itching, eyelid erythema, chemosis, conjunctival congestion, watering, photophobia were reduced when compared to baseline and the improvement was significant in olopatadine group as compared to azelastine group.

Non responder rate was $6.6 \%$ in olopatadine group as compared to $15 \%$ in azelastine group and it was not statistically significant $\left(\chi^{2}=2.15, \mathrm{df}=1, \mathrm{p}=0.1419\right)$, as depicted in Figure 1.

Table 1: Demographic characteristics of the study subjects.

\begin{tabular}{|c|c|c|c|c|}
\hline \multicolumn{2}{|c|}{ Patient characteristics } & Olopatadine group $(n=60)$ & Azelastine group $(n=60)$ & p value \\
\hline \multicolumn{2}{|c|}{ Age in years $($ Mean \pm SD) } & $23.75 \pm 2.98$ & $22.9 \pm 2.74$ & $\mathrm{p}=0.136$ \\
\hline \multicolumn{2}{|l|}{$18-25$ years } & 47 & 43 & \multirow{3}{*}{$\begin{array}{l}\mathrm{p}=0.592 \\
\chi^{2}=1.05, \mathrm{~d} . \mathrm{f}=2\end{array}$} \\
\hline \multicolumn{2}{|l|}{ 26-39 years } & 9 & 10 & \\
\hline \multicolumn{2}{|l|}{$\geq 40$ years } & 4 & 7 & \\
\hline \multirow{2}{*}{ Sex } & Male & 34 & 37 & \multirow{2}{*}{$\begin{array}{l}p=0.577 \\
\chi^{2}=0.310, \text { d.f }=1\end{array}$} \\
\hline & Female & 26 & 23 & \\
\hline \multirow{5}{*}{ Occupation } & Students & 20 & 18 & \multirow{5}{*}{$\begin{array}{l}p=0.914 \\
\chi^{2}=0.972, \text { d. } f=4\end{array}$} \\
\hline & Factory workers & 18 & 19 & \\
\hline & Outdoor workers & 12 & 13 & \\
\hline & Unemployed & 4 & 6 & \\
\hline & Others & 6 & 4 & \\
\hline \multirow{2}{*}{ Habits } & Smokers & 36 & 32 & \multirow{2}{*}{$\begin{array}{l}p=0.461 \\
\chi^{2}=0.543, \text { d.f }=1\end{array}$} \\
\hline & Non-smokers & 24 & 28 & \\
\hline
\end{tabular}

Table 2: Ocular symptoms scores of study subjects.

\begin{tabular}{|c|c|c|c|c|c|c|c|c|}
\hline \multirow{2}{*}{ Ocular symptoms } & \multicolumn{3}{|c|}{ Olopatadine $(n=60)$} & \multicolumn{3}{|c|}{ Azelastine $(n=60)$} & \multicolumn{2}{|c|}{ p value } \\
\hline & Baseline & Visit 1 & Visit 2 & Baseline & Visit 1 & Visit 2 & $\mathrm{p}^{\mathrm{a}}$ & $\mathrm{p}^{\mathrm{b}}$ \\
\hline Itching & $3(2-4)^{£}$ & $2(0-4)^{*}$ & $0(0-4)^{*}$ & $3(2-4)^{\mathfrak{f}}$ & $2(1-4)^{*}$ & $1(0-4)^{*}$ & 0.019 & 0.002 \\
\hline FB sensation & $3(2-4)^{£}$ & $2(1-4)^{*}$ & $0(0-4)^{*}$ & $3(2-4)^{£}$ & $2(1-4) *$ & $1(0-4) *$ & 0.039 & 0.009 \\
\hline Stinging & $3(2-4)^{£}$ & $2(0-4) *$ & $0(0-4)^{*}$ & $3(2-4)^{\mathfrak{f}}$ & $2(0-4) *$ & $1(0-4) *$ & 0.012 & 0.001 \\
\hline photophobia & $3(2-4)^{£}$ & $1(0-4) *$ & $0(0-4)^{*}$ & $3(2-4)^{£}$ & $2(0-4) *$ & $0(0-4) *$ & 0.003 & 0.016 \\
\hline watering & $3(2-4) £$ & $2(0-4) *$ & $0(0-4)^{*}$ & $3(2-4) £$ & $2(0-4) *$ & $1(0-4) *$ & 0.006 & 0.001 \\
\hline
\end{tabular}

scores are depicted in median with range in bracket

*p $<0.0001$, ocular signs scores by F-ANOVA test at visit 1 and visit 2 compared to baseline in both the groups

$£ \mathrm{p}>0.05$, baseline characteristics between groups.

pa - ocular signs scores between groups by Mann - Whitney U test at visit 1

$\mathrm{pb}$ - ocular signs scores between groups by Mann - Whitney U test at visit 2

Table 3: Ocular sign scores of study subjects.

\begin{tabular}{|c|c|c|c|c|c|c|c|c|}
\hline \multirow[t]{2}{*}{ Ocular signs } & \multicolumn{3}{|c|}{ Olopatadine $(n=60)$} & \multicolumn{3}{|c|}{ Azelastine $(n=60)$} & \multicolumn{2}{|c|}{ p value } \\
\hline & Baseline & Visit 1 & Visit 2 & Baseline & Visit 1 & Visit 2 & $\mathrm{p}^{\mathrm{a}}$ & $\mathrm{p}^{\mathrm{b}}$ \\
\hline Congestion & $2(1-3)^{\mathfrak{E}}$ & $1(0-3)^{*}$ & $0(0-3)^{*}$ & $2(1-3)^{£}$ & $1(0-3)^{*}$ & $0(0-3)^{*}$ & 0.038 & 0.008 \\
\hline Erythema & $2(1-3)^{£}$ & $1(0-3)^{*}$ & $0(0-3)^{*}$ & $2(1-3)^{£}$ & $1(0-3)^{*}$ & $1(0-3)^{*}$ & 0.00 & 0.002 \\
\hline Chemosis & $2(1-3)^{£}$ & $1(0-3)^{*}$ & $0(0-3) *$ & $2(1-3)^{£}$ & $2(0-3) *$ & $1(0-3)^{*}$ & 0.046 & 0.015 \\
\hline
\end{tabular}

scores are depicted in median with range in bracket

*p $<0.0001$, ocular signs scores by F-ANOVA test at visit 1 and visit 2 compared to baseline in both the groups

$£ p>0.05$, baseline characteristics between groups.

pa - ocular signs scores between groups by Mann - Whitney U test at visit 1

$\mathrm{pb}$ - ocular signs scores between groups by Mann - Whitney U test at visit 2 


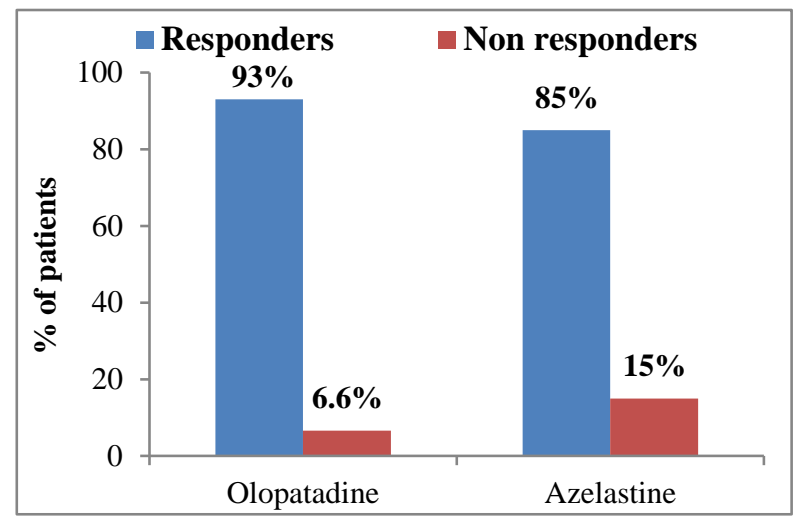

Figure 1: Response rate.

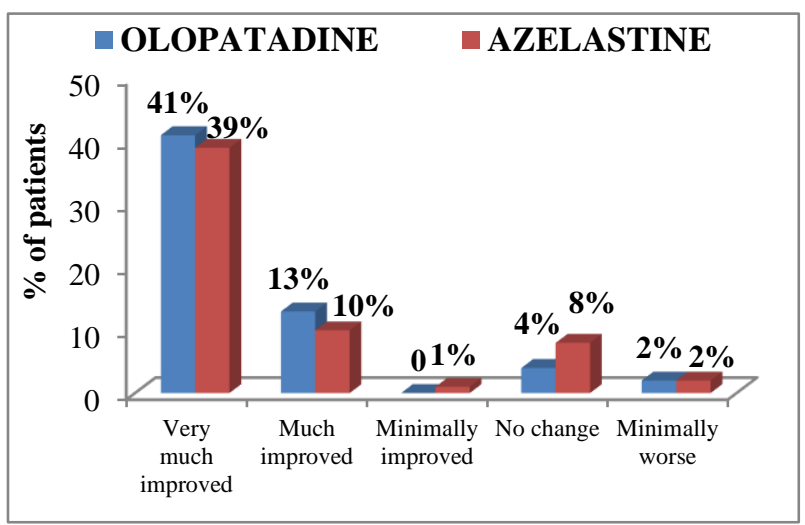

Figure 2: Patient global impression of change in both the groups.

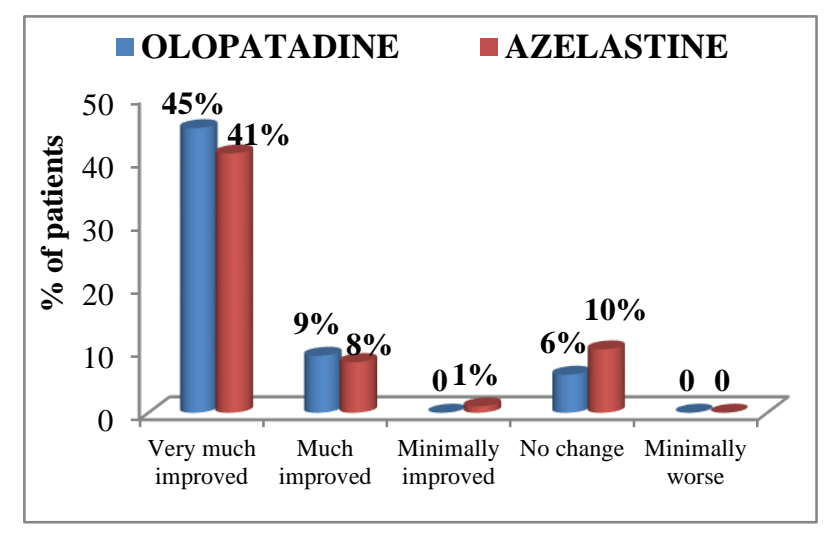

Figure 3: Clinical global impression of change in both the groups.

Table 4: Ocular adverse effects.

\begin{tabular}{|ll|l|}
\hline Adverse effects & $\begin{array}{l}\text { Olopatadine } \\
\text { group } \\
\text { No. of patients: }\end{array}$ & $\begin{array}{l}\text { Azelastine } \\
\text { group }\end{array}$ \\
\hline Stinging & $8(13)$ & $28(47)$ \\
\hline Burning & $9(15)$ & $26(43)$ \\
\hline Headache & $03(5)$ & $02(3)$ \\
\hline Bitter taste & $11(18)$ & $18(30)$ \\
\hline No adverse effect & $32(53)$ & $10(17)$ \\
\hline
\end{tabular}

\section{Side effect and tolerability}

Both the study medications were well tolerated. The Global Evaluation of Tolerability in both the study groups is depicted in Figure 4. All the study participants were $100 \%$ compliant.

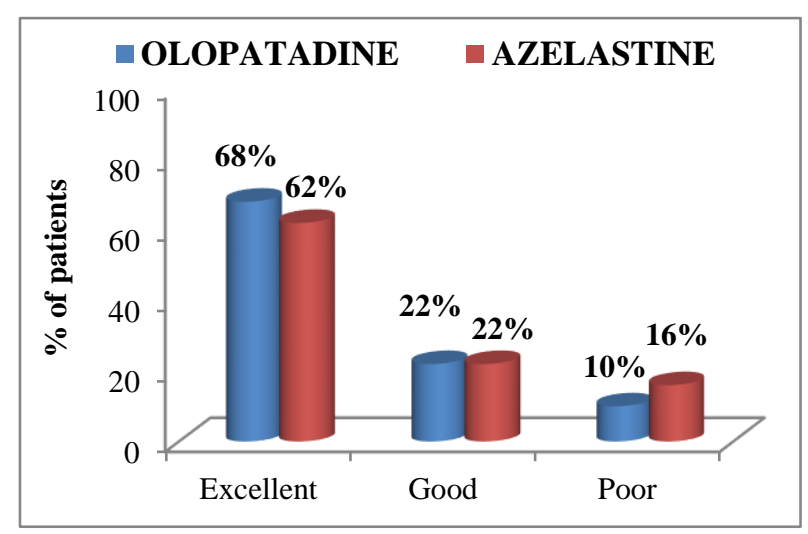

Figure 4: Global evaluation of tolerability in both the groups.

\section{DISCUSSION}

Allergic conjunctivitis is a bilateral and self-limiting inflammatory process caused by an IgE-mediated immediate hypersensitivity reaction triggering mast cell activation and the release of various inflammatory mediators. These contribute to the appearance of the typical signs and symptoms that characterize the disease such as itching, redness, and tearing. ${ }^{14}$ Allergic conjunctivitis affects about $15-20 \%$ of the population worldwide and has a significant impact on the social and economic aspects of life. ${ }^{1-3}$ In the present study the efficacy, safety and tolerability of two topical dual acting anti-allergic drugs, olopatadine and azelastine were compared in patients with allergic conjunctivitis. All the patients recruited in the study were included for analysis as there were no drop outs. Non responders were treated with additional medications such as topical steroids and immunosuppresants. Intention to Treat analysis with Last Observation Carried Forward was done for non responders.

The peak incidence of Allergic conjunctivitis is documented in children and young adults, with no gender predilection. ${ }^{12,15}$ Majority of the patients $(74.1 \%)$ in this study belonged to the 18-25 years age group and only $25.8 \%$ of the patients were above the age of 25 years. This was consistent with the studies done by John et al, and Abokyi et al, where the mean age of allergic conjunctivitis patients was about 20 years. ${ }^{16,17}$ A study done in Spain revealed both children and adults were most commonly affected with allergic conjunctivitis. ${ }^{12}$ However, patients less than 18 years were not included in the present study. In the current study, the two groups were gender matched. Among the subjects enrolled in the two groups there were a total of 71 males and 49 females, 
but this difference too was not statistically significant. A similar trend was also observed in a study done in Greece. However, a cross sectional study done in UAE (76\% females vs $24 \%$ males) revealed female preponderance as compared to males which was statistically significant. ${ }^{16}$

Work-related allergic conjunctivitis has been increasingly reported amongst workers exposed to common environmental allergens. Factory and outdoor workers are at an increased risk of such allergic reactions. ${ }^{18} 31 \%$ and $21 \%$ of the study participants were factory and outdoor workers respectively. This fact is well asserted in a comparative study done in sawmill workers which showed that the incidence of allergic conjunctivitis was more in the technical workers than the administrative workers. ${ }^{19}$

Several eye disorders are seen in smokers and those who are frequently exposed to tobacco smoke. Allergic conjunctivitis is the most common disease affected by environmental variations. Smoking affects the ocular surface, which results in symptoms like itchiness, redness and irritation of eyes. The changes on ocular surface associated with smoking include alteration in lipid layer of tear film, reduced tear secretion and decreased corneal and conjunctival sensitivity. ${ }^{20}$ While no studies have been conducted specifically to assess the relationship between allergic conjunctivitis and tobacco smoke exposure, a number of studies of allergic reactions in children have reported that the risk of developing allergic conjunctivitis is increased by about $20 \%$ in children who are exposed to environmental tobacco smoke. Even in adults smoking has been linked to an increase in ocular surface disorders. $^{21}$ In the present study, $57 \%$ of the allergic conjunctivitis patients were smokers. But to draw a conclusion with this data would not be apt as passive smoking can also increase the risk of these disorders.

In this comparative study, two topical dual acting ocular antihistamines, olopatadine and azelastine, were evaluated for their efficacy and safety in allergic conjunctivitis. Efficacy was evaluated primarily as a reduction in the ocular signs and symptoms scores. The basal ocular signs and symptoms scores were comparable between the two groups. Olopatadine and azelastine, both effectively reduced the scores at the subsequent follow up visits (day 8 and day 15), but the reduction in the olopatadine treated group was significantly larger compared to patients treated with azelastine. At the end of the study period, the reduction in the ocular itching score from baseline was higher in the olopatadine group ( 3 vs 0 ) as compared to the azelastine group (3 vs 1 ). The reduction in ocular congestion score was also higher in the olopatadine group ( 3 vs 0 ) as compared to the azelastine group (3 vs 1). Similarly, the scores of foreign body sensation $(\mathrm{p}<0.009)$, stinging $\quad(\mathrm{p}<0.001)$, photophobia $(\mathrm{p}<0.016)$, tearing $(\mathrm{p}<0.001)$, erythema $(\mathrm{p}<0.002)$ and chemosis $(\mathrm{p}<0.015)$ also showed larger reduction in the olopatadine treated patients compared to the azelastine group. The olopatadine treated patients also showed a lower rate of nonresponders (6.6\%) when compared to patients treated with azelastine $(15 \%)$, though the difference was not statistically significant $\left(\chi^{2}=\right.$ $3.27, \mathrm{df}=1, \mathrm{p}=0.071)$. Hence olopatadine was found to be a more potent and effective dual acting antihistaminic in the treatment of allergic conjunctivitis compared to azelastine.

The findings of this study are consistent with the outcomes noted in the two prospective studies comparing olopatadine and azelastine, the patient-reported PACE study and the investigator-reported CAC study. ${ }^{13,22}$ The recent major multicenter, prospective, open-label PACE (Pataday Allergic Conjunctivitis Evaluation) study conducted by Ophthalmic Research Associates, which included 49 patients of allergic conjunctivitis showed that $42 \%$ of olopatadine treated patients reported an improvement in itching as compared to only $17 \%$ of them treated with azelastine. A reduction in congestion was also reported by a greater number of patients in the olopatadine group (46\%) compared to the azelastine group (20\%). Spangler and colleagues, in the conjunctival allergen challenge (CAC) model, demonstrated that both olopatadine and azelastine were significantly more effective than placebo at reducing itching postchallenge. However, olopatadine was significantly more effective than azelastine in reducing itching at 3.5 minutes postchallenge $(\mathrm{p}<0.05)$. On the contrary, in a survey done at 75 ophthalmologist/ optometrist clinics in South Florida, azelastine was favoured to be more effective and yield higher patient satisfaction score than olopatadine in the treatment of allergic conjunctivitis. ${ }^{23}$

Safety evaluation was another important objective of this study. While $83 \%$ of the patients in azelastine group experienced adverse effects, only $47 \%$ of the patients in olopatadine group reported the same. All the adverse effects encountered were mild to moderate local ocular adverse effects and did not require any medical intervention. There were no serious adverse effects observed in either group. Common adverse effects seen in both the groups were stinging after instillation, burning, bitter taste and headache. Burning (26 vs 9) and stinging (28 vs 8 ) was seen in more number of patients receiving azelastine as compared to those receiving olopatadine which was statistically significant $\left(\chi^{2}=24.9\right.$, $\mathrm{df}=1, \mathrm{p}=0.000$ ). Both the study medications were well tolerated as assessed with the Global Evaluation of Tolerability scale. All the study participants were $100 \%$ compliant. An identical adverse effect and tolerability profile has been observed in various studies conducted with olopatadine and azelastine. The PACE study also reported similar adverse effects with the study medications, however with a lesser incidence rate. The superior comfort with olopatadine compared to azelastine though not been clearly defined, may be at least partly attributed to $\mathrm{pH}$ differences. ${ }^{24}$ Azelastine has a more acidic $\mathrm{pH}$ ranging from 5.0 to 6.5 , whereas olopatadine 
has a physiological $\mathrm{pH}$ of approximately 7. Another possible explanation could be due to the differing effects of these drugs on cell membrane integrity. Olopatadine, unlike azelastine and other dual-action agents, does not perturb the membranes of ocular surface epithelial cells, the damage of which may lead to stinging and burning that is encountered upon instillation of these other agents. $^{25}$

While the strengths of this study include its prospective and randomized study design, an open labelled design remains its limitation. The efficacy and safety of the study medications was also not assessed in the pediatric patients, among whom a higher incidence of allergic conjunctivitis is commonly seen. Further more, elaborate studies evaluating the factors contributing to allergic conjunctivitis, its prognosis and their intervening effects on drug actions may be worth undertaking.

\section{CONCLUSION}

In allergic conjunctivitis, both olopatadine and azelastine were found to be effective in relieving ocular signs and symptoms, but olopatadine was found to be superior in terms of efficacy with minimal side effects.

\section{Funding: No funding sources}

Conflict of interest: None declared

Ethical approval: The study was approved by the Institutional Ethics Committee

\section{REFERENCES}

1. Uchio E. Treatment of allergic conjunctivitis with olopatadine hydrochloride eye drops. Clinical Ophthalmology. 2008;2(3):525-31.

2. Pitt AD, Smith AF, Lindsell L, Voon LW, Rose PW, Bron AJ. Economic and quality of life impact of seasonal allergic conjunctivitis in Oxfordshire. Ophthalmic Epidemiol. Feb 2004;11(1):17-33.

3. Smith AF, Pitt AD, Rodruiguez AE, Alio JL, Marti N, Teus M. The economic and quality of life impact of seasonal allergic conjunctivitis in a Spanish setting. Ophthalmic Epidemiol. Aug 2005;12(4):23342.

4. Irani AM, Butrus SI, Tabbara KF, Schwartz LB. Human conjunctival mast cells: distribution of MCT and MCTC in vernal conjunctivitis and giant papillary conjunctivitis. J Allergy Clin Immunol. 1990;86:34-40.

5. Leonardi A. The role of histamine in allergic conjunctivitis. Acta Ophthalmol Scand. 2000;230:1821.

6. Kidd M, McKenzie SH, Steven I, Cooper C, Lanz R. Efficacy and safety of ketotifen eye drops in the treatment of seasonal allergic conjunctivitis. $\mathrm{Br} \mathrm{J}$ Ophthalmol. Oct 2003;87(10):1206-11.

7. Bielory L, Lien KW, Bigelsen S. Efficacy and tolerability of newer antihistamines in the treatment of allergic conjunctivitis. Drugs. 2005;65(2):21528.
8. Ohmori K. Pharmacological, pharmacokinetic and clinical properties of olopatadine hydrochloride: a new anti-allergic drug. Jpn J Pharmacol. 2002;88:379-97.

9. Ciprandi G, Cosentino C, Milanese M, Tosca MA. Rapid anti-inflammatory action of azelastine eyedrops for ongoing allergic reactions. Ann Allergy Asthma Immunol. 2003;90:434-8.

10. Reddy MS, Suneetha N, Thomas RK, Battu RR. Topical diclofenac sodium for treatment of postoperative inflammation in cataract surgery. Indian journal of ophthalmology. 2000;48(3):223-6.

11. Yaylali V, Demirlenk I, Tatlipinar S, Ozbay D, Esme A, Yildirim C, et al. Comparative study of $0.1 \%$ olopatadine hydrochloride and $0.5 \%$ ketorolac tromethamine in the treatment of seasonal allergic conjunctivitis. Acta Ophthalmol. Scand. 2003;81:378-82.

12. Rosario N, Bielory L. Epidemiology of allergic conjunctivitis. Curr Opin Allergy Clin Immunol. 2011;11:471.

13. Spangler DL, Bensch G, Berdy GJ. Evaluation of the efficacy of olopatadine hydrochloride $0.1 \%$ ophthalmic solution and azelastine hydrochloride $0.05 \%$ ophthalmic solution in the conjunctival allergen challenge model. Clin Ther. 2001 Aug;23(8):1272-80.

14. Sánchez MC, Parra BF, Matheu V, Navarro A, Ibáñez MD, Dávila I, et al. Allergic conjunctivitis (SEAIC Rhinoconjuntivitis Committee 2010). J Investig Allergol Clin Immunol. 2011;21(2):1-19.

15. Singh K, Axelrod S, Bielory L. The epidemiology of ocular and nasal allergy in the United States, 19881994. J Allergy Clin Immunol. 2010;126(4):778.

16. John J, Ahmed S, Anjum F, Kebab M, Mohammed N, Darwich H. Prevalence of Allergies among University Students: A Study from Ajman, United Arab Emirates. ISRN Allergy. 2014:502052.

17. Abokyi S, Koffuor G, Ntodie M, Kyei S, Gyanfosu L. Epidemiological profile and pharmacological management of allergic conjunctivitis: A study in Ghana. Int J Pharm. 2012;3(4):195-201.

18. Peate WF. Work-Related Eye Injuries and Illnesses. Am Fam Physician 2007;75:1017-22.

19. Njinaka I, Uhumwangho M, Edema TO, Dawodu OA, Omoti AE. A Comparison Study of Conjunctiva Disorders in Technical and Administrative Sawmill Workers in Nigeria. Malaysian $\mathrm{J}$ Med Sci. 2011;18(3):43-8.

20. Lois N, Abdelkader E, Reglitz K, Garden C, Ayres J G. Environmental tobacco smoke exposure and eye disease. Br J Ophthalmol. 2008;92(10):1304-10.

21. Shubhrica. Effect of Environment on Eyes: A Review. Indian Journal of Clinical Practice. Sept 2013;24(4):381-4.

22. Epstein AB, Hoven TV, Kaufman A, Carr W. Management of allergic conjunctivitis: an evaluation of the perceived comfort and therapeutic efficacy of olopatadine $0.2 \%$ and azelastine $0.05 \%$ from two prospective studies. Clin Ophthalmol. 2009;3:329-36. 
23. Oliva AM, Slonim CB. Patient Evaluation of Azelastine $\mathrm{HCl}$ versus Olopatadine $\mathrm{HCl}$ in the Treatment of Allergic Conjunctivitis. Investigative Ophthalmology and Visual Science. May 2005;46:937.

24. Abelson MB, Loeffler O. Conjunctival allergen challenge: models in the investigation of ocular allergy. Curr Allergy Asthma Rep. 2003;3:363-8.

25. Brockman HL, Momsen MM, Knudtson JR, Miller ST, Graff G, Yanni JM. Interactions of olopatadine and selected antihistamines with model and natural membranes. Ocul Immunol Inflamm. 2003;11(4):247-68.

Cite this article as: Jayanthi CR, Nanthini R, Vijayalakshmi. A prospective study of efficacy and safety of olopatadine versus azelastine in allergic conjunctivitis at a tertiary care hospital. Int J Basic Clin Pharmacol 2017;6:1948-54. 